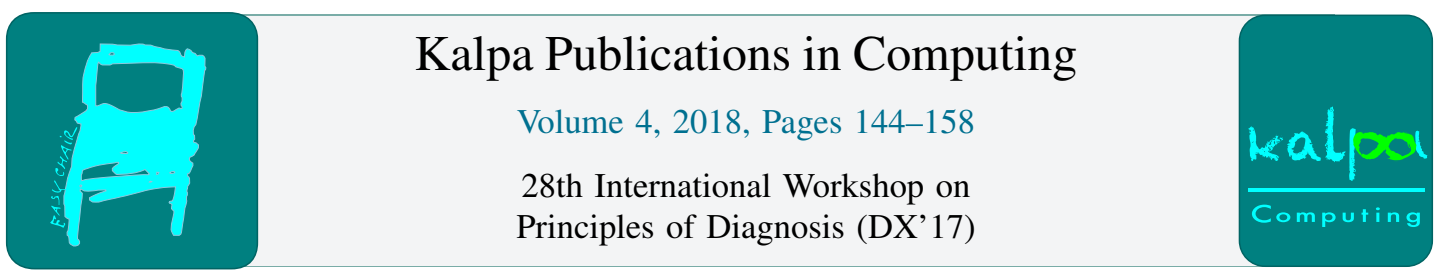

\title{
Decentralized Diagnosis of Discrete Event Systems Using an Arborescent Architecture
}

\author{
Ahmed Khoumsi ${ }^{1}$ \\ Department of Electrical \& Computer Engineering University of Sherbrooke, Canada \\ ahmed. khoumsi@usherbrooke.ca
}

\begin{abstract}
Decentralized diagnosis of discrete event systems consists in detecting faults in discrete event systems by using decentralized architectures. In particular, inference-based diagnosis is a decentralized architecture of interest, since it is more general than several other decentralized architectures. In this paper, we first propose a method that realizes a diagnosis objective $\mathcal{D}$ by an arborescent architecture (or tree). Each leaf of the tree is a decentralized diagnosis, and each node $n$ is a disjunction or conjunction of the diagnosis decisions of the two children of $n$. Then, we show that if inference-based diagnosis is applicable to $\mathcal{D}$, then all the leafs of the obtained tree are basic decentralized diagnosers. This implies that every inference-based diagnosis is realizable by a combination of basic decentralized diagnosers.
\end{abstract}

\section{Introduction}

The objective of diagnosis is to detect faults (or failures) in the behavior of a plant modeled as a discrete event system (DES). [1, 2, 3] are among the pioneering studies on diagnosis of DES. Several studies have followed, like $[4,5,6,7,8,9,10,11,12]$. Some authors study the diagnosis of specific DES, for example decentralized or distributed diagnosis [13,14, 15, 16, 17, 18, 19, 20, 21, 22, 23, 24], and diagnosis with timing requirements [25, 8, 26, 27, 28, 10, 29].

The study of this paper is in the category of decentralized diagnosis, where several local diagnosers observe the plant and cooperate to generate decisions. A decision is a verdict of the presence or absence of fault. The authors of $[14,19]$ propose decentralized diagnosis architectures consisting of simple diagnosers to detect the presence or absence of faults. They also propose more elaborate and general diagnosers that generate conditional decisions. The authors of $[17,18,23]$ propose inferencebased diagnosis that generalizes significantly several existing architectures, e.g. those of $[14,19]$. In inference-based diagnosis, each decision made by a local diagnoser is associated to an ambiguity level, and the global decision actually made is the local decision with the smallest ambiguity level. Another particularity of inference-based diagnosis, is that three decisions are possible, instead of only two: 0 , for the presence of fault; 1 , for the absence of fault; and $\phi$ when the diagnoser cannot determine if a fault has occurred or not. In several previous studies, 0 and $\phi$ are not distinguished.

We propose a procedure that, given a decentralized diagnosis objective $\mathcal{D}$, constructs an arborescent architecture (or tree) that realizes $\mathcal{D}$. Each leaf of the tree represents a decentralized diagnosis architecture, and each node $n$ is a disjunction or conjunction of the decisions of the two children of $n$. 
We consider the case where $\mathcal{D}$ is realizable by inference-based diagnosis and show that in the obtained tree, every leaf is a basic decentralized diagnoser, i.e. a diagnoser of the simplest category of decentralized diagnosers. Therefore, every inference-based diagnosis (which may be nonintuitive and difficult to apprehend) is realizable by a combination of basic decentralized diagnosers.

The arborescent architecture has recently been proposed in supervisory control [30]. Despite the analogy between supervisory control and diagnosis, the adaptation of [30] to diagnosis is not an easy task because there exist fundamental differences between the two areas.

The rest of the paper is organized a follows. In Sect. 2, we introduce diagnosis of DES with emphasis on inference-based diagnosis. Sect. 3 presents the three basic decentralized diagnosers used in our proposition. Sect. 4 presents the methodology to construct an arborescent architecture realizing a given diagnosis objective $\mathcal{D}$. In Sect. 5, we study the tree construction when inference-based diagnosis is applicable to $\mathcal{D}$. In Section 6, we conclude our study.

For lack of space, the proofs of our results (propositions, lemmas and theorems) are omitted.

\section{Diagnosis of Discrete Event Systems}

\subsection{Generalities on Discrete Event Systems}

Alphabet denotes a finite set of discrete events, trace denotes a finite sequence of discrete events, and language denotes a set of traces. $\varepsilon$ denotes the empty trace, and $|\lambda|$ denotes the length of a trace $\lambda$. If $\Sigma$ is an alphabet, $\Sigma^{*}$ denotes the (infinite) language containing all the traces constructed from events of $\Sigma$. Consider two traces $\lambda$ and $\mu$ s.t. $\lambda=\mu \alpha$ for some trace $\alpha: \mu$ is said a prefix of $\lambda$, and $\lambda$ is said a postfix of $\mu . \bar{L}$ denotes the set of prefixes of a language $L$. $L$ is said prefix-closed if $L=\bar{L}$. Given two languages $L$ and $K, L \backslash K$ is the language obtained by removing from $L$ all the traces of $K . P_{i}(L)$ and $P_{i}^{-1}(L)$ denote the usual projection and inverse projection of a language $L$ in an alphabet $\Sigma_{i}$.

A discrete event system (DES) is modeled by a regular language containing all the traces it can execute. Therefore, a DES is also modeled by a finite state automaton $(\mathrm{FSA}) \mathcal{A}=\left(Q, \Sigma, \delta, q_{0}\right)$, where $Q$ is a finite set of states, $q_{0} \in Q$ is the initial state, $\Sigma$ is an alphabet, and the transitions are specified by a partial function $\delta: Q \times \Sigma \rightarrow Q$. The language of $\mathcal{A}$ contains the traces leading from $q_{0}$ to the states in $Q$.

\subsection{Introduction to Diagnosis}

Consider a DES, called plant, modeled by a FSA $\mathcal{A}=\left(Q, \Sigma, \delta, q_{0}\right)$, and let $\mathcal{L}$ be the (prefix-closed) language of $\mathcal{A}$. Some events in $\Sigma$ model the occurrence of faults (or failures). For our study, we consider only one of these events, which is noted $f$. When there are several faulty events that must be distinguished, the same study must be repeated for each faulty event. $\mathcal{L}$ is partitioned in two languages: $\mathcal{H}$ contains the traces of $\mathcal{L}$ without $f$, and $\mathcal{F}$ contains the traces of $\mathcal{L}$ with at least one occurrence of $f$. Formally, $\mathcal{F}=\left\{\lambda \in \mathcal{L} \mid \exists u, v \in \Sigma^{*}, \lambda=u f v\right\}$, and $\mathcal{H}=\mathcal{L} \backslash \mathcal{F}$. We also define $\mathcal{F}^{m}=\{\lambda \in \mathcal{F} \mid \exists u, v \in$ $\left.\Sigma^{*},|v| \geq m, \lambda=u f v\right\} \subseteq \mathcal{F}$, which contains the traces with a fault followed by at least $m$ events. Note that $\mathcal{F} \cup \mathcal{H}=\mathcal{L}, \mathcal{F} \cap \mathcal{H}=\emptyset$, and $\mathcal{F}^{0}=\mathcal{F}$. $\mathcal{H}$ is prefix-closed, which means that if a trace is non-faulty then its "past" (i.e. all its prefixes) is non-faulty. $\mathcal{F}$ is postfix-closed, i.e. $\mathcal{F} \Sigma^{*} \cap \mathcal{L}=\mathcal{F}$, which means that if a trace is faulty then its "future" (i.e. all its postfixes) is faulty.

Several variants of diagnosis objectives can be found in the literature. We adopt the objective of [17, 23]. A diagnoser observes the behavior of the plant and takes one of the following decisions: 1 (resp. 0 ) when it is certain that $f$ has (resp. has not) occurred, and $\phi$ when it is uncertain whether $f$ has occurred or not. $f$ is unobservable for the diagnoser, otherwise the diagnosis is trivial. Let $\operatorname{Diag}(\lambda) \in\{1,0, \phi\}$ 
be the decision made by the diagnoser when the plant has executed a trace $\lambda \in \mathcal{L}$. The objective is to respect Eqs. $(1,2,3)[17,23]$ :

$$
\begin{gathered}
\exists m \geq 0 \text { s.t. }: \forall \lambda \in \mathcal{F}^{m}, \operatorname{Diag}(\lambda)=1 \\
\forall \lambda \in \mathcal{F}, \operatorname{Diag}(\lambda) \neq 0 \\
\forall \lambda \in \mathcal{H}, \operatorname{Diag}(\lambda) \neq 1
\end{gathered}
$$

Eq. (1) means that a fault occurrence is detected after a bounded number of events, and Eqs. $(2,3)$ mean that the decisions are not erroneous.

Eqs. $(1,2)$ are with respect to (or w.r.t) $\mathcal{F}$, and Eq. (3) is w.r.t $\mathcal{H}$. We can also say that Eqs. $(1,2,3)$ are w.r.t $(\mathcal{F}, \mathcal{H})$. This remark is relevant, because in our proposition we will decompose $\mathcal{F}$ and $\mathcal{H}$, and thus we will have to define Eqs. $(1,2,3)$ w.r.t $(\mathcal{U}, \mathcal{V})$, where $\mathcal{U} \subset \mathcal{F}$ and $\mathcal{V} \subset \mathcal{H}$.

We make the following assumptions [2]:

A1 There is a transition at each state of $\mathcal{A}$.

A2 Every cycle of $\mathcal{A}$ contains at least one event observable at by the diagnoser.

A1 is used for simplicity and can be easily relaxed [2]. A2 is necessary to detect faults within bounded delays.

To every diagnosis architecture $X$, are associated a property called $X$-diagnosability and a category of diagnosers called $X$-diagnosers, s.t. the following equivalence is respected: there exists an $X$-diagnoser satisfying Eqs. $(1,2,3)$ w.r.t $(\mathcal{F}, \mathcal{H})$ iff $(\mathcal{F}, \mathcal{H})$ is $X$-diagnosable.

In decentralized diagnosis, the most generally adopted model is that $n$ local diagnosers $\left(\mathcal{D}_{i}\right)_{i \in I}$ ( $I=\{1,2, \cdots, n\})$ observe the plant and take local decisions based on their observations; a global decision is generated by combining in a certain way the $n$ local decisions. Each $\mathcal{D}_{i}$ has a partial view of the plant, i.e. it observes only the events that belongs to some $\Sigma_{i} \subset \Sigma$.

The following proposition will be necessary to clarify certain aspects of our arborescent methodology:

Proposition 1. We have the following two properties:

1. $\mathcal{F} \cap\left[\bigcap_{i \in I} P_{i}^{-1} P_{i}(\mathcal{H})\right] \neq \emptyset$,

2. $\mathcal{H} \cap\left[\bigcap_{i \in I} P_{i}^{-1} P_{i}(\mathcal{F})\right] \neq \emptyset$.

These two properties are deduced from the fact that for every trace $\lambda \in \mathcal{H}$ s.t. $\lambda f \in \mathcal{F}$, we have $\lambda \in \mathcal{H} \cap\left[\bigcap_{i \in I} P_{i}^{-1} P_{i}(\mathcal{F})\right]$ and $\lambda f \in \mathcal{F} \cap\left[\bigcap_{i \in I} P_{i}^{-1} P_{i}(\mathcal{H})\right]$. These two properties are relevant from the fact that in our arborescent methodology, we decompose $\mathcal{F}$ and $\mathcal{H}$ (respecting the two properties), which implies the use of pairs $(\mathcal{U}, \mathcal{V})$, where $\mathcal{U} \subseteq \mathcal{F}$ and $\mathcal{V} \subseteq \mathcal{H}$. This will justify the use of two specific basic diagnosers: F-diagnoser and NF-diagnoser, which are applied for pairs $(\mathcal{U}, \mathcal{V})$ not satisfying Property 1 and Property 2, respectively.

\subsection{Inference-Based Diagnosis}

Inference-based diagnosis of [23] is based on the following iterative languages $\mathcal{F}[k]$ and $\mathcal{H}[k]$ which are also the basis of our arborescent methodology: $\mathcal{F}[0]=\mathcal{F}, \mathcal{H}[0]=\mathcal{H}$, and for $k \geq 0: \mathcal{F}[k+1]=$ $\mathcal{F}[k] \cap\left[\bigcap_{i \in I} P_{i}^{-1} P_{i}(\mathcal{H}[k])\right]$ and $\mathcal{H}[k+1]=\mathcal{H}[k] \cap\left[\bigcap_{i \in I} P_{i}^{-1} P_{i}(\mathcal{F}[k])\right]$. 
Proposition 2. Here are some properties of $\mathcal{F}[k]$ and $\mathcal{H}[k]$ :

1. $\mathcal{F}[k+1] \subseteq \mathcal{F}[k]$ and $\mathcal{H}[k+1] \subseteq \mathcal{H}[k] \forall k \geq 0$.

2. If $\mathcal{F}[k+1]=\mathcal{F}[k]$ and $\mathcal{H}[k+1]=\mathcal{H}[k]$ for some $k \geq 0$, then $\mathcal{F}[i]=\mathcal{F}[k]$ and $\mathcal{H}[i]=\mathcal{H}[k] \forall i \geq k$.

3. If $\mathcal{F}[k]=\emptyset$ for some $k \geq 0$, then $\mathcal{F}[i]=\mathcal{H}[i]=\emptyset \forall i>k$.

4. If $\mathcal{F}[k+2]=\mathcal{F}[k]$ for some $k \geq 0$, then $\mathcal{F}[i]=\mathcal{F}[k]$ and $\mathcal{H}[i+1]=\mathcal{H}[k+1] \forall i \geq k$.

Properties 3 and 4 also hold if $\mathcal{F}$ and $\mathcal{H}$ are switched.

In inference-based diagnosis [23], when the plant has executed a trace $\lambda \in \mathcal{L}$, every $\mathcal{D}_{i}$ has observed $P_{i}(\lambda)$ and computes a pair $\operatorname{Diag}_{i}\left(P_{i}(\lambda)\right)=\left(d_{i}\left(P_{i}(\lambda)\right), n_{i}\left(P_{i}(\lambda)\right)\right)$, where $d_{i}\left(P_{i}(\lambda)\right) \in\{1,0, \phi\}$ is a local decision, and $n_{i}\left(P_{i}(\lambda)\right)$ is a nonnegative integer representing an ambiguity level. The global decision $\operatorname{Diag}(\lambda)$ is the local decision with the smallest ambiguity level. $d_{i}\left(P_{i}(\lambda)\right)$ and $n_{i}\left(P_{i}(\lambda)\right)$ are computed in a rigorous way, which is however quite complex and nonintuitive. We do not present such computation which is not indispensable for understanding arborescent framework. Let us however present some notions and results.

An $N$-Inferring diagnoser is a specific diagnoser used in inference-based diagnosis whose maximum ambiguity level is $N$, and $N$-Inference F-diagnosability is the corresponding diagnosability. The authors of [23] prove that $N$-Inference F-diagnosability is necessary and sufficient for the existence of an $N$ Inferring diagnoser that respects Eqs. $(1,2,3)$. They also prove that $N$-Inference F-diagnosability generalizes the diagnosabilities defined in $[14,16,19]$. More precisely, they prove that: $\operatorname{Inf} f_{0}$-F-diagnosability 0 -Inference F-diagnosability is equivalent to codiagnosability of [16], and $\operatorname{Inf}_{1}$-F-diagnosability 1Inference F-diagnosability implies COND-F-CODIAG of [14, 19]. We complete the comparison by:

Proposition 3. 0-Inference F-diagnosability is equivalent to F-CODIAG of [14, 19].

\section{Basic Diagnosers That Constitute The Tree}

As mentioned in Sect. 2, our arborescent methodology is based on decomposing the diagnosis of $(\mathcal{F}, \mathcal{H})$ into a combination of diagnoses of several pairs, where each pair $(\mathcal{U}, \mathcal{V})$ is s.t. $\mathcal{U} \subseteq \mathcal{F}$ and $\mathcal{V} \subseteq \mathcal{H}$. Depending on characteristics of each pair $(\mathcal{U}, \mathcal{V})$, it is diagnosed by one of the three basic decentralized diagnosers: $\operatorname{Inf} f_{0}$-F-diagnoser, F-diagnoser or NF-diagnoser that we present in this section. These are the only diagnosers that are used in our arborescent methodology. By diagnosing a pair $(\mathcal{U}, \mathcal{V})$, we mean observing the behavior of the plant and making decisions $(1,0, \phi)$ that satisfy Eqs. $(1,2,3)$ w.r.t $(\mathcal{U}, \mathcal{V})$, instead of w.r.t $(\mathcal{F}, \mathcal{H})$. Therefore, the decisions of each diagnoser will be computed as functions of $(\mathcal{U}, \mathcal{V})$, instead of $(\mathcal{F}, \mathcal{H})$. Henceforth, the fact that $\mathcal{U} \subseteq \mathcal{F}$ and $\mathcal{V} \subseteq \mathcal{H}$ is implicit.

As we will see, $\operatorname{Inf} f_{0}$-F-diagnoser is applied to pairs $(\mathcal{U}, \mathcal{V})$ respecting the two properties of Prop. 1, while $\mathrm{F}$-diagnoser and NF-diagnoser are respectively applied to pairs $(\mathcal{U}, \mathcal{V})$ not respecting properties 1 and 2 of Prop. 1.

\section{1 $\operatorname{Inf}_{0}$-F-diagnoser}

The local decisions $\left(\operatorname{Diag}_{i}\left(P_{i}(\lambda)\right)\right)_{i \in I}$ and the global decision $\operatorname{Diag}(\lambda)$ of $\operatorname{Inf}_{0}$-F-diagnoser are computed as follows:

$$
\operatorname{Diag}_{i}\left(P_{i}(\lambda)\right)=\left\{\begin{array}{ll}
1, & \text { if } P_{i}(\lambda) \in P_{i}(\mathcal{U}) \backslash P_{i}(\mathcal{V}), \\
0, & \text { if } P_{i}(\lambda) \in P_{i}(\mathcal{V}) \backslash P_{i}(\mathcal{U}), \\
\phi, & \text { otherwise. }
\end{array}\right\}
$$




$$
\operatorname{Diag}(\lambda)=\left\{\begin{array}{c}
1, \text { if } \exists i \in I \text { s.t. } \operatorname{Diag}_{i}\left(P_{i}(\lambda)\right)=1 \text { and } \\
\forall i \in I, \operatorname{Diag}_{i}\left(P_{i}(\lambda)\right) \neq 0 \\
0, \text { if } \exists i \in I \text { s.t. } \operatorname{Diag}_{i}\left(P_{i}(\lambda)\right)=0 \text { and } \\
\forall i \in I, \operatorname{Diag}_{i}\left(P_{i}(\lambda)\right) \neq 1 \\
\phi, \text { otherwise. }
\end{array}\right\}
$$

We have the following definition and theorem:

Definition 1. $(\mathcal{U}, \mathcal{V})$ is said to be $\operatorname{Inf}_{0}-F$-diagnosable if $\exists m \geq 0$ s.t. $\mathcal{U}^{m} \cap\left[\bigcap_{i \in I} P_{i}^{-1} P_{i}(\mathcal{V})\right]=\emptyset$.

Theorem 1. Inf $f_{0}-F$-diagnoser satisfies Eqs. $(1,2,3)$ w.r.t $(\mathcal{U}, \mathcal{V})$ iff $(\mathcal{U}, \mathcal{V})$ is Inf $_{0}-F$-diagnosable.

Note that if we take $(\mathcal{U}, \mathcal{V})=(\mathcal{F}, \mathcal{H})$, we have exactly the results of [23] for an ambiguity level $N=0$, i.e. the $\operatorname{Inf} f_{0}$-F-diagnoser is a 0 -inferring diagnoser, and $I n f_{0}$-F-diagnosability is the 0 -Inference F-diagnosability of [23].

\subsection{F-diagnoser}

The local decisions $\left(\operatorname{Diag}_{i}\left(P_{i}(\lambda)\right)\right)_{i \in I}$ and the global decision $\operatorname{Diag}(\lambda)$ of F-diagnoser are computed as follows:

$$
\begin{gathered}
\operatorname{Diag}_{i}\left(P_{i}(\lambda)\right)=\left\{\begin{array}{ll}
0 & \text { if } P_{i}(\lambda) \in P_{i}(\mathcal{V}), \\
1 & \text { if } P_{i}(\lambda) \notin P_{i}(\mathcal{V}) .
\end{array}\right\} \\
\operatorname{Diag}(\lambda)=\bigvee_{i \in I} \operatorname{Diag}_{i}\left(P_{i}(\lambda)\right)
\end{gathered}
$$

We define strong F-diagnosability as follows:

Definition 2. $(\mathcal{U}, \mathcal{V})$ is said to be strongly $F$-diagnosable if $\mathcal{U} \cap\left[\bigcap_{i \in I} P_{i}^{-1} P_{i}(\mathcal{V})\right]=\emptyset$.

Note that strong F-diagnosability of $(\mathcal{U}, \mathcal{V})$ implies that $(\mathcal{U}, \mathcal{V})$ does respect the first property of Prop. 1. We have the following theorem:

Theorem 2. If $(\mathcal{U}, \mathcal{V})$ is strongly $F$-diagnosable, then $F$-diagnoser satisfies Eqs. $(1,2,3)$ w.r.t $(\mathcal{U}, \mathcal{V})$.

We have the following proposition which justifies the use of the qualifier strong:

Proposition 4. Strong F-diagnosability implies F-CODIAG of [14, 19], codiagnosabibility of [16] and Inf $_{0}$-F-diagnosability of [23].

\subsection{NF-diagnoser}

The local decisions $\left(\operatorname{Diag}_{i}\left(P_{i}(\lambda)\right)\right)_{i \in I}$ and the global decision $\operatorname{Diag}(\lambda)$ of NF-diagnoser are computed as follows:

$$
\begin{gathered}
\operatorname{Diag}_{i}\left(P_{i}(\lambda)\right)=\left\{\begin{array}{ll}
1 & \text { if } P_{i}(\lambda) \in P_{i}(\mathcal{U}), \\
0 & \text { if } P_{i}(\lambda) \notin P_{i}(\mathcal{U}) .
\end{array}\right\} \\
\operatorname{Diag}(\lambda)=\bigwedge_{i \in I} \operatorname{Diag}_{i}\left(P_{i}(\lambda)\right)
\end{gathered}
$$

We define the following strong NF-diagnosability: 
Definition 3. $(\mathcal{U}, \mathcal{V})$ is said strongly $N F$-diagnosable if $\mathcal{V} \cap\left[\bigcap_{i \in I} P_{i}^{-1} P_{i}(\mathcal{U})\right]=\emptyset$.

Note that strong NF-diagnosability of $(\mathcal{U}, \mathcal{V})$ implies that $(\mathcal{U}, \mathcal{V})$ does respect the second property of Prop. 1. We have the following theorem:

Theorem 3. If $(\mathcal{U}, \mathcal{V})$ is strongly NF-diagnosable, then NF-diagnoser satisfies Eqs. $(1,2,3)$ w.r.t $(\mathcal{U}, \mathcal{V})$.

We have the following proposition which justifies the use of the qualifier strong:

Proposition 5. Strong NF-diagnosability implies NF-CODIAG of [14, 19] and 0-Inference NFdiagnosability of [18].

\section{Synthesis of an Arborescent Architecture}

We will see that our arborescent methodology is based on decomposing iteratively and alternately $\mathcal{F}$ and $\mathcal{H}$. Throughout the iterative process, the current pair to be decomposed is $(\mathcal{U}, \mathcal{V})$ where $\mathcal{U} \subseteq \mathcal{F}$ and $\mathcal{V} \subseteq \mathcal{H}$. For the purpose of our methodology, we define the iterative languages $\mathcal{U}[k]$ and $\mathcal{V}[k]$ like $\mathcal{F}[k]$ and $\mathcal{H}[k]$ have been defined in Sect. 2.3.

In Sect. 4.2, we will decompose the diagnosis of $(\mathcal{U}, \mathcal{V})$ into a conjunction of two diagnoses inspired from the multi-decision diagnosis [24]. With a similar approach, in Sect. 4.3 we will decompose the diagnosis of $(\mathcal{U}, \mathcal{V})$ into a disjunction of two diagnoses. We will use the expression "to realize the diagnosis of $(\mathcal{U}, \mathcal{V})$ ", which means: to generate diagnosis decisions respecting Eqs. $(1,2,3)$ w.r.t $(\mathcal{U}, \mathcal{V})$.

\subsection{Series $\left(r_{i}\right)_{i \geq 1}$ and $\left(s_{i}\right)_{i \geq 1}$}

In order to present clearly our methodology, we need to define two series $\left(r_{i}\right)_{i \geq 1}$ and $\left(s_{i}\right)_{i \geq 1}$ and their respective indexes $\nu$ and $\mu$ :

- $r_{1}=(\mathcal{F}, \mathcal{H}), r_{2 k+2}=(\mathcal{F}[2 k+1], \mathcal{H}[2 k])$ and $r_{2 k+3}=(\mathcal{F}[2 k+1], \mathcal{H}[2 k+2])$ for $k \geq 0$.

Let $r_{\nu}$ be the first element of $\left(r_{i}\right)_{i \geq 1}$ that has a component $\mathcal{F}[j]$ s.t. $\mathcal{F}[j] \cap \mathcal{F}^{m}=\emptyset$ for some $m \geq 0$, if any.

- $s_{1}=(\mathcal{F} \backslash \mathcal{F}[1], \mathcal{H}), s_{2 k+2}=(\mathcal{F}[2 k+1], \mathcal{H}[2 k] \backslash \mathcal{H}[2 k+2])$ and $s_{2 k+3}=(\mathcal{F}[2 k+1] \backslash \mathcal{F}[2 k+$ 3], $\mathcal{H}[2 k+2])$ for $k \geq 0$.

Let $s_{\mu}$ be the first element of $\left(s_{i}\right)_{i \geq 1}$ that has an empty component $\mathcal{F}[j] \backslash \mathcal{F}[j+2]$ or $\mathcal{H}[j] \backslash \mathcal{H}[j+2]$, if any.

\subsection{Conjunctive Diagnosis of $(\mathcal{U}, \mathcal{V})$ by Decomposing $\mathcal{V}$}

Consider two values $d 1$ and $d 2$ in the set $\{1,0, \phi\}$. The conjunctive combination of $d 1$ and $d 2$, denoted $d 1 \wedge d 2$, is defined as follows:

$$
d 1 \wedge d 2=\left\{\begin{array}{ll}
1, & \text { if } d 1=1 \text { and } d 2=1, \\
0, & \text { if } d 1=0 \text { or } d 2=0 \\
\phi, & \text { for " } 1 \wedge \phi " \text { and " } \phi \wedge 1 " .
\end{array}\right\}
$$

Consider a decomposition of $\mathcal{V}$ into $\mathcal{V} 1$ and $\mathcal{V} 2$, i.e. $\mathcal{V}=\mathcal{V} 1 \cup \mathcal{V} 2$. Let the conjunctive diagnosis of $(\mathcal{U}, \mathcal{V} 1)$ and $(\mathcal{U}, \mathcal{V} 2)$ consist in combining conjunctively the decisions of the diagnoses of $(\mathcal{U}, \mathcal{V} 1)$ and $(\mathcal{U}, \mathcal{V} 2)$. The following proposition is deduced from [24]:

Proposition 6. The conjunctive diagnosis of $(\mathcal{U}, \mathcal{V} 1)$ and $(\mathcal{U}, \mathcal{V} 2)$ realizes the diagnosis of $(\mathcal{U}, \mathcal{V} 1 \cup \mathcal{V} 2)$. 
By applying Prop. 6 to $r_{2 k+2}=(\mathcal{F}[2 k+1], \mathcal{H}[2 k])$, with a decomposition of $\mathcal{H}[2 k]$ into $\mathcal{H}[2 k] \backslash$ $\mathcal{H}[2 k+2]$ and $\mathcal{H}[2 k+2]$, we obtain the following proposition:

Proposition 7. The diagnosis of $r_{2 k+2}$ is realized by the conjunctive diagnosis of $s_{2 k+2}$ and $r_{2 k+3}$, for $k \geq 0$.

The following proposition implies that every $s_{2 k+2}$ can be diagnosed by a NF-diagnoser:

Proposition 8. $s_{2 k+2}$ is strongly NF-diagnosable, for $k \geq 0$.

Prop. 7 and Prop. 8 are illustrated in Fig. 1.

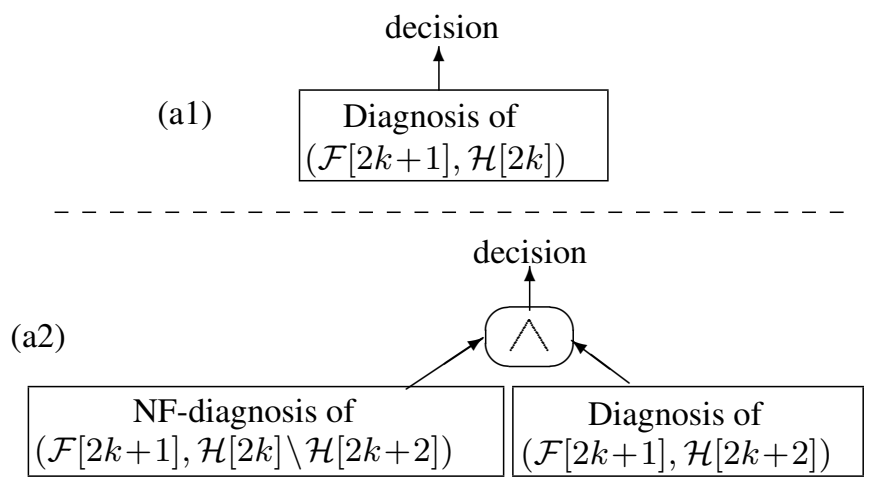

Figure 1: Decomposition of $\mathcal{V}$ : (a1) realized by (a2).

\subsection{Disjunctive Diagnosis of $(\mathcal{U}, \mathcal{V})$ by Decomposing $\mathcal{U}$}

Consider two values $d 1$ and $d 2$ in the set $\{1,0, \phi\}$. The disjunctive combination of $d 1$ and $d 2$, denoted $d 1 \vee d 2$, is defined as follows:

$$
d 1 \vee d 2=\left\{\begin{array}{ll}
0, & \text { if } d 1=0 \text { and } d 2=0, \\
1, & \text { if } d 1=1 \text { or } d 2=1, \\
\phi, & \text { for " } 0 \wedge \phi " \text { and " } \phi \wedge 0 " .
\end{array}\right\}
$$

Consider a decomposition of $\mathcal{U}$ into $\mathcal{U} 1$ and $\mathcal{U} 2$, i.e. $\mathcal{U}=\mathcal{U} 1 \cup \mathcal{U} 2$. Let the disjunctive diagnosis of $(\mathcal{U} 1, \mathcal{V})$ and $(\mathcal{U} 2, \mathcal{V})$ consist in combining disjunctively the decisions of the diagnoses of $(\mathcal{U} 1, \mathcal{V})$ and $(\mathcal{U} 2, \mathcal{V})$. We have the following proposition:

Proposition 9. The disjunctive diagnosis of $(\mathcal{U} 1, \mathcal{V})$ and $(\mathcal{U} 2, \mathcal{V})$ realizes the diagnosis of $(\mathcal{U} 1 \cup \mathcal{U} 2, \mathcal{V})$.

By applying Prop. 9 to $r_{2 k+1}$ for $k \geq 0$, we obtain the following proposition:

Proposition 10. The diagnosis of $r_{2 k+1}$ is realized by the disjunctive diagnosis of $s_{2 k+1}$ and $r_{2 k+2}$, for $k \geq 0$.

The following proposition implies that every $s_{2 k+1}$ can be diagnosed by a F-diagnoser:

Proposition 11. $s_{2 k+1}$ is strongly $F$-diagnosable, for $k \geq 0$.

Prop. 10 and Prop. 11 are illustrated in Figs. 2 and 3, where Fig. 2 corresponds to the diagnosis of $r_{1}$, and Fig. 3 corresponds to the diagnosis of $r_{2 k+3}$ for $k \geq 0$. 


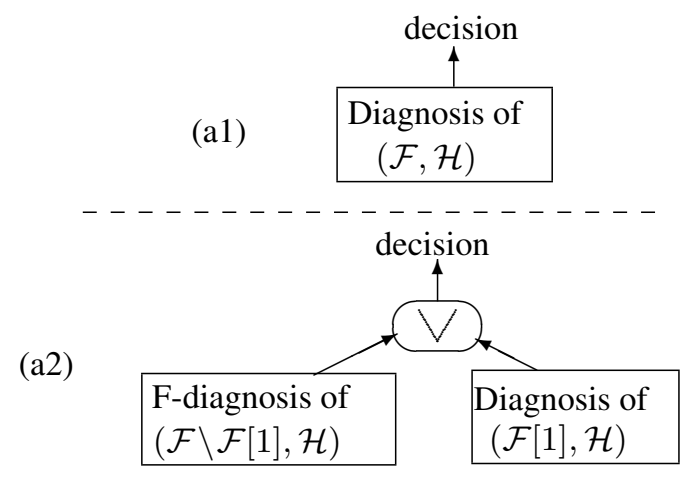

Figure 2: Decomposition of $\mathcal{U}$ : (a1) realized by (a2).

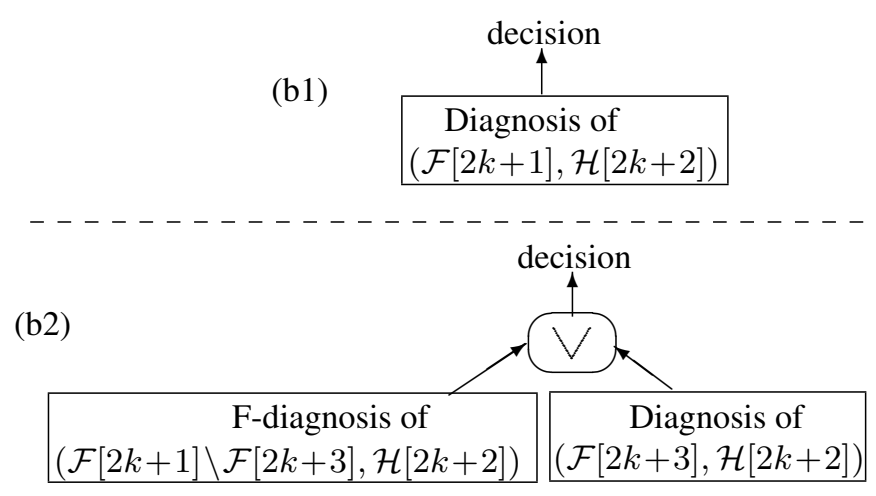

Figure 3: Decomposition of $\mathcal{U}$ : (b1) realized by (b2).

\subsection{Tree Construction}

We construct an arborescent architecture to diagnose $(\mathcal{F}, \mathcal{H})$; the construction method is based on Props. 7, 8, 10 and 11, which are illustrated in Figs. 1, 2 and 3. Firstly, the objective of diagnosing $r_{1}=\left(\mathcal{F}, \mathcal{H}\right.$ ) is split disjunctively (as shown in Fig. 2) into the diagnoses of $s_{1}$ (left child) and $r_{2}$ (right child). The latter diagnosis of $r_{2}$ is itself split conjunctively (as shown in Fig. 1 for $k=0$ ) into the diagnoses of $s_{2}$ (left child) and $r_{3}$ (right child). The latter diagnosis of $r_{3}$ is itself split disjunctively (as shown in Fig. 3 for $k=0$ ) into the diagnoses of $s_{3}$ (left child) and $r_{4}$ (right child). And so on, decompositions with conjunctive or disjunctive combination are applied alternately to the successive $r_{i}$, until we obtain a $r_{i}$ whose right child $r_{i+1}$ respects $C 1_{i+1}$ or left child $s_{i}$ respects $C 2_{i}$; such $r_{i}$ is a leaf.

$C 1_{i}$ : for $r_{i}=(\mathcal{F}[u], \mathcal{H}[v]), \exists m \geq 0$ s.t. $\mathcal{F}[u] \cap \mathcal{F}^{m}=\emptyset$.

$C 2_{2}: s_{i}$ has an empty component $\mathcal{H}[j] \backslash \mathcal{H}[j+2]$ or $\mathcal{F}[j] \backslash \mathcal{F}[j+2]$

Notations 1. Let $x$ and $y$ be pairs in the form $(\mathcal{U}, \mathcal{V})$ to be diagnosed:

- $\langle x\rangle$ denotes a leaf representing the diagnosis of $x$;

- $\langle x\rangle_{\mathrm{F}}$ and $\langle x\rangle_{\mathrm{NF}}$ denote leafs representing the F-diagnosis and NF-diagnosis of x, respectively;

- $\langle x\rangle_{\mathrm{F}} \vee\langle y\rangle$ is a node $\vee$ with the left child $\langle x\rangle_{\mathrm{F}}$ and the right child $\langle y\rangle$; 
- $\langle x\rangle_{\mathrm{NF}} \wedge\langle y\rangle$ is a node $\wedge$ with the left child $\langle x\rangle_{\mathrm{NF}}$ and the right child $\langle y\rangle$.

The tree (which we denote $\mathcal{T}$ ) is constructed as follows:

- Initialization: $\mathcal{T}=\left\langle r_{1}\right\rangle=(\mathcal{F}, \mathcal{H}) ; \quad i=1$.

- While none of $C 1_{i+1}$ and $C 2_{i}$ is respected:

- if $i$ is odd: replace $\left\langle r_{i}\right\rangle$ by $\left\langle s_{i}\right\rangle_{\mathrm{F}} \vee\left\langle r_{i+1}\right\rangle$;

- if $i$ is even: replace $\left\langle r_{i}\right\rangle$ by $\left\langle s_{i}\right\rangle_{\mathrm{NF}} \wedge\left\langle r_{i+1}\right\rangle$;

$-i=i+1$.

The obtained tree is represented in Fig. 4. This arborescent architecture realizes the diagnosis of $(\mathcal{F}, \mathcal{H})$. Note that in this tree, $\mathcal{F}[j]$ and $\mathcal{H}[j]$ use odd and even indexes, respectively. Each node $n$ of the tree has two children: the left child of $n$ is a leaf which provides the decisions of a F-diagnoser or NF-diagnoser, and the right child of $n$ is a subtree which provides the decisions of a diagnosis.

Note that $\left(r_{i}\right)_{i \geq 1}$ are the pairs represented at the upper right sides of the nodes of Fig. 4. The successive $r_{i}$ are diagnosed by the successive nested subtrees. Note also that $\left(s_{i}\right)_{i \geq 1}$ are the pairs represented in the left leafs of Fig. 4. The successive $s_{i}$ are diagnosed by the successive left leafs. The fact that each left child (which is a leaf) of a node is a NF-diagnoser or F-diagnoser is due to Props. 8 and 11 .

The tree of Fig. 4 has been obtained by starting with a disjunction and a decomposition of $\mathcal{F}$. If we start with a conjunction and a decomposition of $\mathcal{H}$, we obtain a symmetrical tree, i.e. each of the two trees is obtained from the other tree by making the following switches: $\mathcal{F}[j] \leftrightarrow \mathcal{H}[j], \vee \leftrightarrow \wedge$, F-diagnosis $\leftrightarrow$ NF-diagnosis. Consequently, the results obtained from the two trees are symmetrical. Therefore, we will consider only the tree of Fig. 4. However, we will explain later that in some examples, only one of the two trees is constructible.

We see that in the construction of the tree, the while-loop stops when $C 1_{i+1}$ or $C 2_{i}$ is respected. The case where the loop stops due to $C 1_{i+1}$ is studied in Sect. 5, while the case where the loop stops due to $C 2_{i}$ is left for a future study.

The reader might ask the following two questions, to which we provide some explanations:

- Why do we use $\mathcal{F}[j] \backslash \mathcal{F}[j+2]$ and $\mathcal{H}[j] \backslash \mathcal{H}[j+2]$ in $\left(s_{i}\right)_{i \geq 1}$, instead of $\mathcal{F}[j] \backslash \mathcal{F}[j+1]$ and $\mathcal{H}[j] \backslash \mathcal{H}[j+1]$ ?

This choice is due to Prop. 2(4), which guarantees that before the convergence of $(\mathcal{F}[j])_{j>0}$ and $(\mathcal{H}[j])_{j \geq 0}$, we have $\mathcal{F}[j] \backslash \mathcal{F}[j+2] \neq \emptyset$ and $\mathcal{H}[j] \backslash \mathcal{H}[j+2] \neq \emptyset$, respectively. This result will be useful in Sect. 5.

- Why do we distinguish even and odd indices ?

This is because the series $\left(r_{i}\right)_{i \geq 1}$ and $\left(s_{i}\right)_{i \geq 1}$ have distinct forms with even and odd indices.

\section{Arborescence of Basic Diagnoses to Realize an Inference-Based Diagnosis}

\subsection{Obtained Tree}

In this section, we make the following assumption, which means that $(\mathcal{F}, \mathcal{H})$ is $\operatorname{Inf}_{N}$-F-diagnosable and implies that inference-based diagnosis is applicable to $(\mathcal{F}, \mathcal{H})$ [23]: 


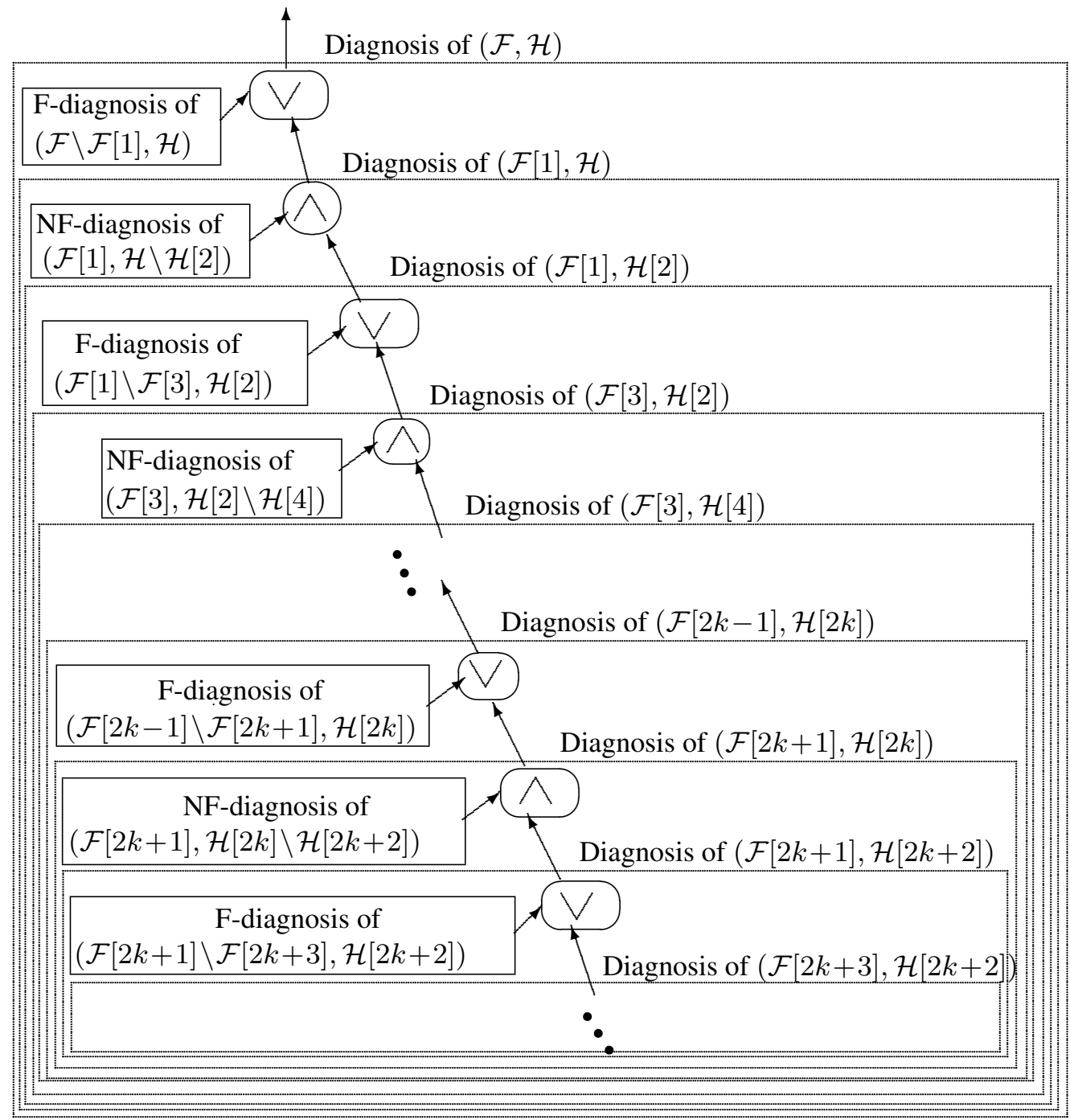

Figure 4: Tree obtained if we start with a disjunction.

Assumption 1. $\exists N, m \geq 0$ s.t. $\mathcal{F}[N+1] \cap \mathcal{F}^{m}=\emptyset$.

The tree of Fig. 4 is constructible iff $\mathcal{F} \neq \mathcal{F}[1]$, because $\mathcal{F}$ is initially decomposed into $\mathcal{F} \backslash \mathcal{F}[1]$ and $\mathcal{F}[1]$. Similarly, its symmetrical tree (introduced above) is constructible iff $\mathcal{H} \neq \mathcal{H}[1]$, because $\mathcal{H}$ is initially decomposed into $\mathcal{H} \backslash \mathcal{H}[1]$ and $\mathcal{H}[1]$. Therefore, none of the two trees is constructible iff $\mathcal{F}=\mathcal{F}[1]$ and $\mathcal{H}=\mathcal{H}[1]$. Let us show that this case is impossible. From Prop. 2(2), it implies $\mathcal{F}[k]=\mathcal{F}$ and $\mathcal{H}[k]=\mathcal{H} \forall k \geq 0$, and hence $\mathcal{F}[N+1] \cap \mathcal{F}^{m}=\mathcal{F} \cap \mathcal{F}^{m}=\mathcal{F}^{m}$ which, from Assump. 1, must be empty for some $m \geq 0$. This is contradictory with Assumption A1 of Sect. 2.2 which implies that $\mathcal{F}^{m} \neq \emptyset \forall m \geq 0$. Therefore, Assump. 1 implies that at least one of the two trees is constructible. Due to the symmetry between the two trees, it is sufficient to present only the study of the tree of Fig. 4, which we will do in this section, assuming $\mathcal{F} \neq \mathcal{F}[1]$. We have the following lemma: 
Lemma 1. Assump. 1 is equivalent to the existence of an even $\nu \geq 2$ s.t. $r_{\nu}$ is the first element of $\left(r_{i}\right)_{i \geq 1}$ whose component $\mathcal{F}[j]$ respects $\mathcal{F}[j] \cap \mathcal{F}^{m}=\emptyset$ for some $m \geq 0$.

In the remaining part of this Sect. 5, Assump. 1 is implicit when $\nu$ is mentioned. We have the following lemma:

Lemma 2. $s_{1}, \cdots, s_{\nu-1}$ do not contain an empty component.

In the construction of the tree, $i=\nu-1$ is the first index where the while-loop has its condition unsatisfied. More precisely, for $i=\nu-1, C 1_{i+1}$ is true (from Lemma 1) and $C 2_{i}$ is false (from Lemma 2). Therefore, the last iteration is for $i=\nu-2$, and hence $s_{\nu-2}$ and $r_{\nu-1}$ are the last constructed elements.

We have the following proposition which implies that $r_{\nu-1}$ is diagnosable by the $\operatorname{Inf} f_{0}$-F-diagnoser of Sect. 3.1.

Proposition 12. $r_{\nu-1}$ is Inf $f_{0}-F$-diagnosable.

We deduce the following proposition:

Proposition 13. If $\nu=2$, Inf $f_{0}-F$-diagnosis is applicable to $r_{1}=(\mathcal{F}, \mathcal{H})$, hence the tree that realizes the diagnosis of $(\mathcal{F}, \mathcal{H})$ consists of just one leaf. If $\nu=2 k+4$ for $k \geq 0$, the tree of Fig. 5 realizes the diagnosis of $(\mathcal{F}, \mathcal{H})$.

As already explained, the tree of Fig. 4 is constructible iff $\mathcal{F} \neq \mathcal{F}[1]$, and a symmetrical tree is constructible iff $\mathcal{H} \neq \mathcal{H}[1]$. Prop. 13 has been obtained from the tree of Fig. 4, and a similar result can be obtained from the symmetrical tree. Since Assump. 1 implies that $\mathcal{F} \neq \mathcal{F}[1]$ or $\mathcal{H} \neq \mathcal{H}[1]$, at least one of the two trees is constructible, hence:

Theorem 4. Every inference-based diagnosis of $(\mathcal{F}, \mathcal{H})$ is equivalent to an arborescent architecture consisting of disjunctions and conjunctions of F-diagnoses and NF-diagnoses and a unique $\operatorname{Inf} f_{0}-F$ diagnosis.

\subsection{Example}

Let us illustrate our results with the example of [23], so that the reader has the same example to compare our arborescent diagnosis with inference-based diagnosis of [23]. We consider the automaton of Fig. 6, where the initial state is numbered 0 , the states in bold are reached by $\mathcal{F}$, and the other states are reached by $\mathcal{H}$. We have $n=2, I=\{1,2\}, \Sigma_{1}=\left\{a, a^{\prime}, c, d\right\}$ and $\Sigma_{2}=\left\{b, b^{\prime}, c, d\right\}$. We compute:

- $\mathcal{F}=c^{*}\left(a f c^{*}+b f c^{*}+d\left(f c^{*}+a f\left(\varepsilon+b^{\prime} c^{*}\right)+b f\left(\varepsilon+a^{\prime} c^{*}\right)\right)\right), \mathcal{H}=\overline{c^{*}\left(a b^{\prime} c^{+}+b a^{\prime} c^{+}+d\left(a c^{+}+b c^{+}\right)\right)}$,

- $\mathcal{F}[1]=c^{*}\left(a f c^{*}+b f c^{*}+d\left(f c^{*}+a f+b f\right)\right), \mathcal{H}[1]=\overline{c^{*}\left(a+b+d\left(a c^{+}+b c^{+}\right)\right)}$,

- $\mathcal{F}[2]=c^{*}\left(a f+b f+d\left(f c^{*}+a f+b f\right)\right)$,

- $\mathcal{F}[i]=c^{*}(a f+b f+d(f+a f+b f))$, for $i \geq 3, \mathcal{H}[i]=\overline{c^{*}(a+b+d(a+b))}$, for $i \geq 2$,

$\nu=4$ because $r_{4}=(\mathcal{F}[3], \mathcal{H}[2])$ is the first $r_{i}$ that has its component $\mathcal{F}[j]$ s.t. $\mathcal{F}[j] \cap \mathcal{F}^{m}=\emptyset$ for some $m \geq 0$. Indeed, we have $\mathcal{F}[3] \cap \mathcal{F}^{1}=\emptyset$, and $d f c^{m} \in \mathcal{F}[2] \cap \mathcal{F}^{m} \neq \emptyset$ for every $m \geq 1$. We are in the case $\nu=2 k+4$ of Prop. 13 with $k=0$. We obtain therefore the tree of Fig. 7, which corresponds to Fig. 5 for $k=0$. The local and global decisions taken by this architecture are represented in Table 1 . where $X, Y, Z$ are the global decisions indicated in Fig. 7. 


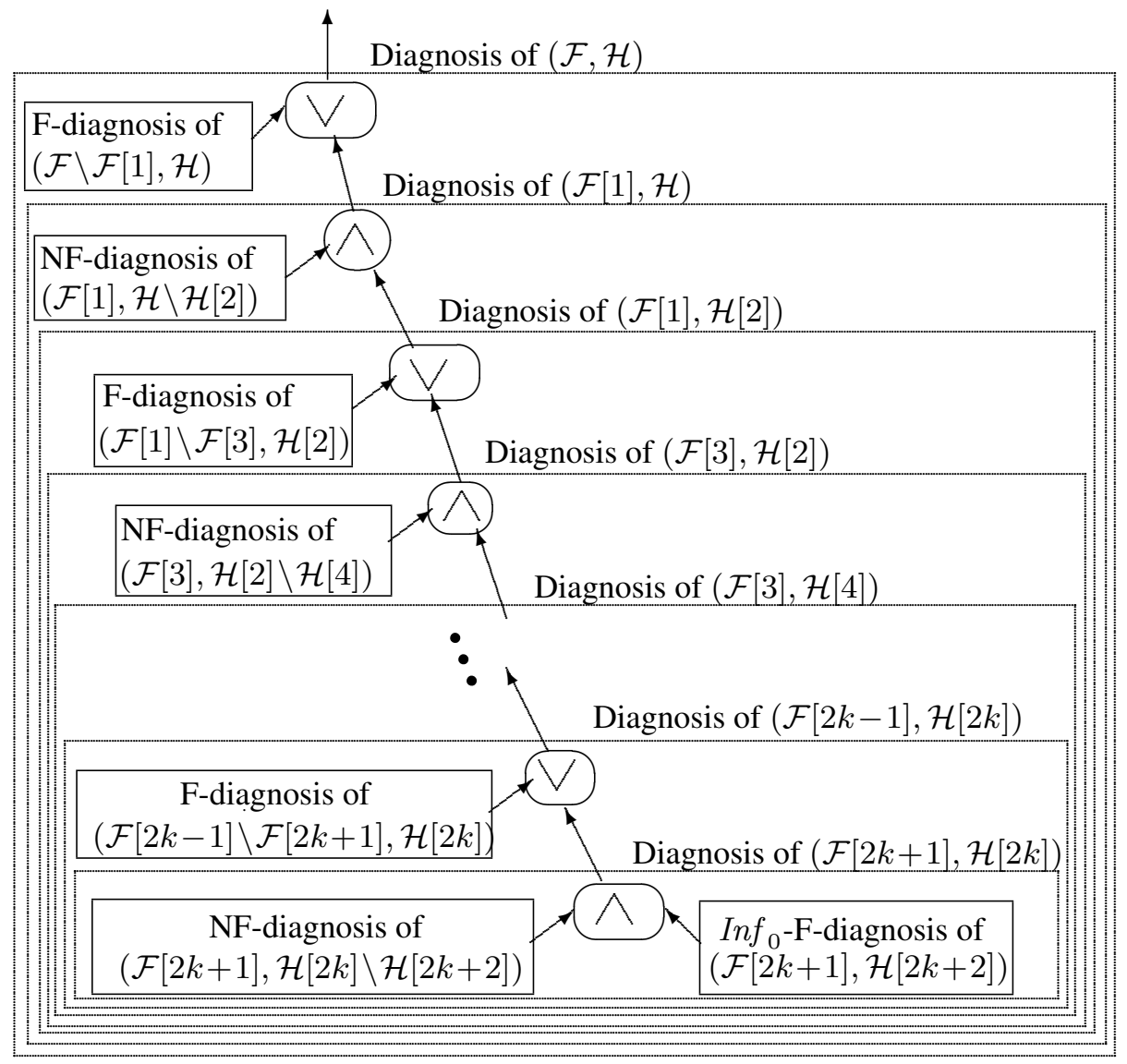

Figure 5: Tree of Fig. 4 when $\nu=2 k+4$ for $k \geq 0$.

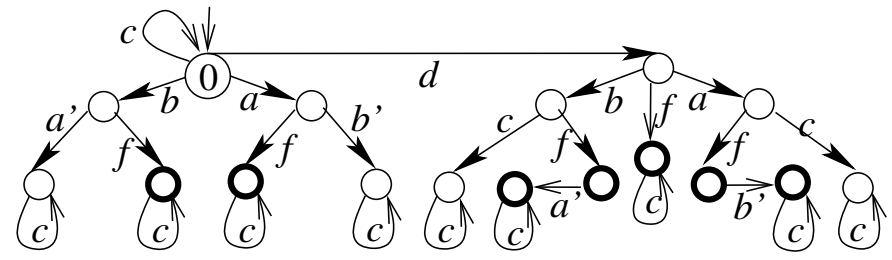

Figure 6: Example: the states in bold are reached by $\mathcal{F}$, and the other states are reached by $\mathcal{H}$.

\section{Conclusion}

An arborescent architecture for decentralized diagnosis of discrete event systems is proposed. We show that when inference-based diagnosis is applicable to realize a given diagnosis objective $\mathcal{D}$, the latter is also realizable by an arborescent architecture combining uniquely the most basic decentralized diagnosers. This result shows that inference-based diagnosis is fundamentally based on basic decentralized diagnosers.

We are now investigating the case where inference-based diagnosis is unapplicable to realize $\mathcal{D}$. 


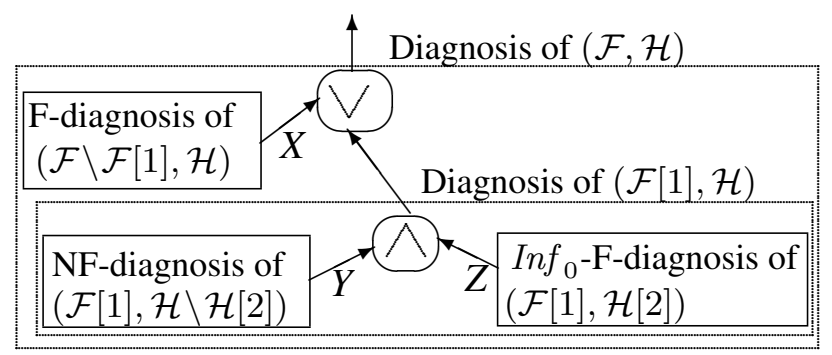

Figure 7: Arborescent architecture of the example of Fig. 6.

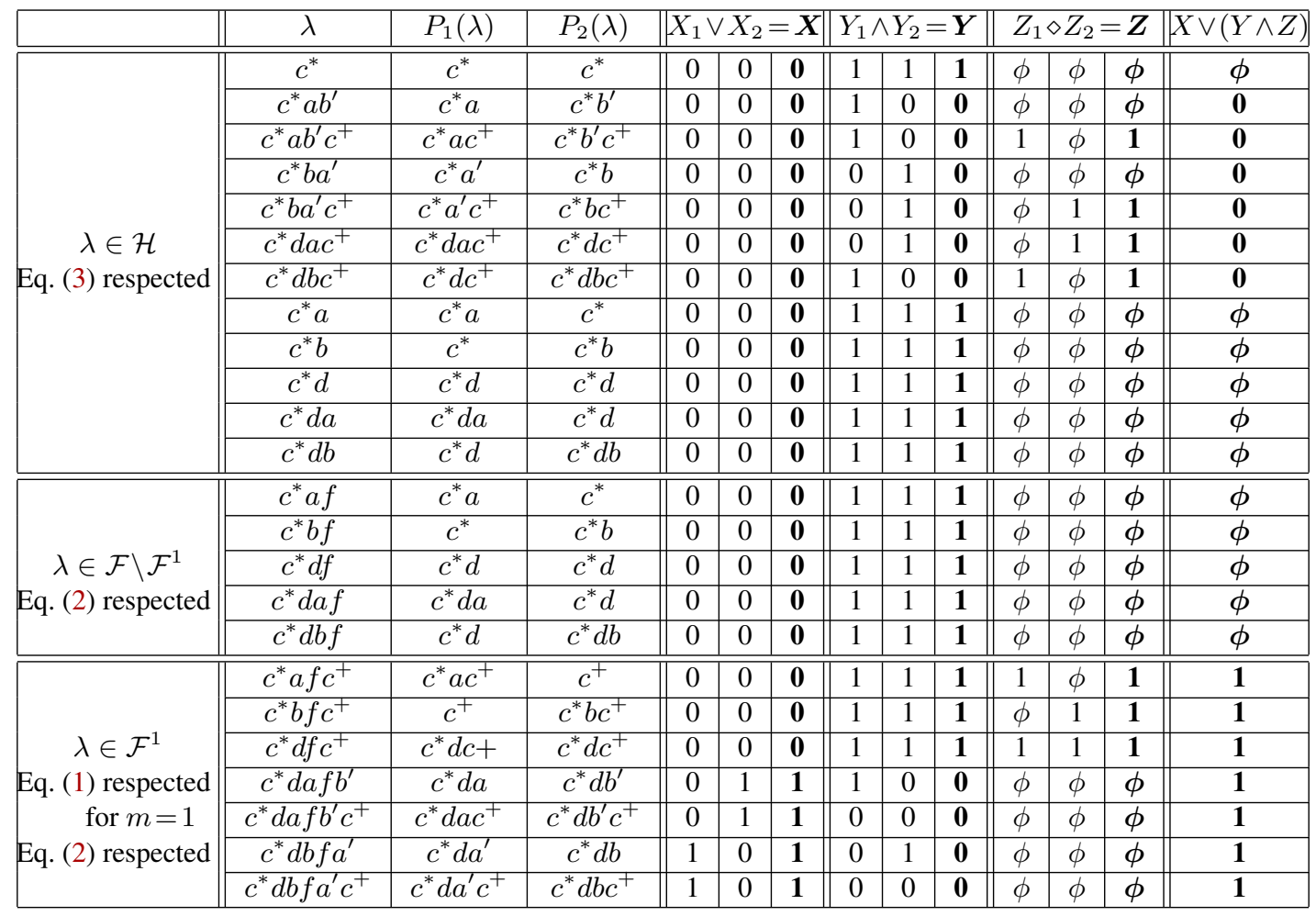

Table 1: Local and global decisions taken by the arborescent architecture of Fig. 7. The operator $\diamond$ corresponds to Eq. (5). 
In this case, we obtain a tree whose leafs are basic decentralized diagnosers, with the exception of the lower right leaf. The latter corresponds to a diagnosis objective $\lfloor\mathcal{D}\rfloor$ where inference-based diagnosis is unapplicable. Therefore, the problem of finding a diagnosis architecture for $\mathcal{D}$ is reduced into the problem of finding a diagnosis architecture for $\lfloor\mathcal{D}\rfloor$. The latter can be interpreted as the portion of $\mathcal{D}$ which is responsible of the nonapplicability of inference-based diagnosis. This fact is relevant for multidecision diagnosis [24] that has to find how to decompose a diagnosis objective which is unrealizable by inference-based diagnosis, into several diagnosis objectives that are realizable by inference-based diagnosis. Instead of finding a decomposition of $\mathcal{D}$, now multi-decision diagnosis has to find a decomposition of $\lfloor\mathcal{D}\rfloor$.

\section{References}

[1] F. Lin. Diagnosability of discrete event systems and its applications. Discrete Event Dyna. Syst.: Theory Applicat., 4(2):197-212, 1994.

[2] M. Sampath, R. Sengupta, S. Lafortune, K. Sinnamohidden, and D. Teneketzis. Diagnosability of discrete event systems. IEEE Transactions on Automatic Control, 40:1555-1575, Sept. 1995.

[3] M. Sampath, R. Sengupta, S. Lafortune, K. Sinnamohideen, and D. Teneketzis. Failure Diagnosis Using Discrete Event Models. IEEE Transactions on Control Systems Technolog, 4(2), March 1996.

[4] R. Sengupta. Diagnosis and communication in distributed systems. In Intern. Workshop on Discrete Event Systems (WODES), pages 144-151, Cagliari, Italy, August 1998.

[5] S. Jiang, Z. Huang, V. Chandra, and R. Kumar. A Polynomial Time Algorithm for Diagnosability of Discrete Event Systems. IEEE Transactions on Automatic Control, 46(8):1318-1321, 2001.

[6] T.S. Yoo and S. Lafortune. Polynomial-time verification of diagnosability of partially observed discrete-event systems. IEEE Transactions on Automatic Control, 47(9):1491-1495, 2002.

[7] S.H. Zad, R.H. Kwong, and W.M. Wonham. Fault Diagnosis in Discrete-Event Systems: Framework and Model Reduction. IEEE Transactions on Automatic Control, 48(7):1199-1212, 2003.

[8] S. Jiang, Z. Huang, V. Chandra, and R. Kumar. Failure Diagnosis of Discrete-Event Systems with Linear-Time Temporal Logic Specifications. IEEE Transactions on Automatic Control, 49(6):934-945, 2004.

[9] G. Vachtsevanos, F. Lewis, M. Roemer, A. Hess, and B. Wu. Intelligent Fault Diagnosis and Prognosis for Engineering Systems. Hoboken, NJ: John Wiley and Sons, 2006.

[10] S. Biswas, D. Sarkara, P. Bhowala, and S. Mukhopadhyay. Diagnosis of Delay-Deadline Failures in Real Time Discrete Event Models. ISA Transactions, 46(4):569-582, October 2007.

[11] F. Cassez and S. Tripakis. Fault Diagnosis with Static and Dynamic Observers. Fundamenta Informaticae, 88(4):497-540, December 2008.

[12] J. Dubreil, T. Jéron, and H. Marchand. Monitoring Confidentiality by Diagnosis Techniques. In European Control Conference (ECC), Budapest, Hungary, August 2009.

[13] R. Debouk, S. Lafortune, and D. Teneketzis. Coordinated decentralized protocols for failure diagnosis of discrete event systems. Discrete Event Dyna. Syst.: Theory Applicat., 10:33-86, 2000.

[14] Y. Wang, T. Yoo, and S. Lafortune. Decentralized Diagnosis of Discrete Event Systems Using Unconditional and Conditional Decisions. In Conference on Decision and Control (CDC), Seville, Spain, December 2005.

[15] S. Lafortune, Y. Wang, and T. Yoo. Diagnostic décentralisé des systèmes à événements discrets. In 5th Colloque Francophone sur la Modélisation des Systèmes Réactifs (MSR), Grenoble, France, October 2005.

[16] R. Kumar and S. Takai. Decentralized Failure Diagnosis of Discrete Event Systems. IEEE Transactions on Systems, Man, and Cybernetics, A, 36(2):384Ü-395, 2006.

[17] R. Kumar and S. Takai. Inference-Based Ambiguity Management in Decentralized Decision-Making : Decentralized Diagnosis of Discrete Event Systems. In American Control Conference (ACC), Minneapolis, Minnesota, USA, June 2006.

[18] S. Takai and R. Kumar. Decentralized Diagnosis for Nonfailures of Discrete Event Systems Using InferenceBased Ambiguity Management. In 8th Intern. Workshop on Discrete Event Systems (WODES), Ann Arbor, 
Michigan, USA, July 2006.

[19] Y. Wang, T. Yoo, and S. Lafortune. Diagnosis of Discrete Event Systems Using Decentralized Architectures. Discrete Event Dynamic Systems: Theory and Practice, 17(2):233-263, 2007.

[20] A. Khoumsi and H. Chakib. A Multi-Decision Approach for Decentralized Diagnosis of the Presence and Absence of Faults in Discrete Event Systems. In 16th Mediterranean Conf. on Control and Automation (MED), Ajaccio, Corsica, France, June 2008.

[21] S. Takai and R. Kumar. Inference-Based Decentralized Prognosis in Discrete Event Systems. In Conference on Decision and Control (CDC), Cancun, Mexico, December 2008.

[22] C. Chakib and A. Khoumsi. Multi-decision Diagnosis: Parallel Decentralized Architectures Cooperating for Diagnosing Discrete Event Systems. In European Control Conference (ECC), Budapest, Hungary, August 2009.

[23] R. Kumar and S. Takai. Inference-Based Ambiguity Management in Decentralized Decision-Making: Decentralized Diagnosis of Discrete Event Systems. IEEE Transactions on Automation Science and Engineering, 6(3):479-491, 2009.

[24] H. Chakib and A. Khoumsi. Multi-Decision Diagnosis: Decentralized Architectures Cooperating for Diagnosing the Presence of Faults in Discrete Event Systems. Discrete Event Dynamic Systems (JDEDS), 2012.

[25] S. Tripakis. Fault Diagnosis for Timed Automata. In Formal Techniques in Real-Time and Fault-Tolerant Systems (FTRTFT), LNCS 2469, pages 205-221. Springer-Verlag, 2002.

[26] P. Bouyer, F. Chevalier, and D. D'Souza. Fault Diagnosis Using Timed Automata. In 8th Intern. Conf. on Foundations of Software Science and Computation Structures (FoSSaCS), Edinburgh, Scotland, 2005.

[27] S.H. Zad, R.H. Kwong, and W.M. Wonham. Fault Diagnosis in Discrete-Event Systems: Incorporating Timing Information. IEEE Transactions on Automatic Control, 50(7):1010-1015, 2005.

[28] S. Jiang and R. Kumar. Diagnosis of Dense-Time Systems Using Digital Clocks. In American Control Conference (ACC), June 2006.

[29] A. Khoumsi and L. Ouédraogo. Diagnosis of Faults in Real-Time Discrete Event Systems. In IFAC Symposium on Fault Detection, Supervision, and Safety of Technical Processes, Barcelona, Spain, June-July 2009.

[30] A. Khoumsi and H. Chakib. Arborescent architecture for decentralized supervisory control of discrete event systems. In International Conference on Control, Decision and Information Technologies (CoDIT), Barcelona, Spain, April 2017.

http://www.gel.usherbrooke.ca/khoumsi/Research/Public/CoDIT2017.pdf. 